\title{
Alcohol consumption and COVID-19 severity: a propensity score matched study in China
}

\author{
Lin Lv ${ }^{1,2, \dagger}$, Yi-Wu Zhou ${ }^{1,2, \dagger}$, Rong Yao ${ }^{1,2, *}$
}

\author{
${ }^{1}$ Department of Emergency Medicine, \\ Emergency Medical Laboratory, West \\ China Hospital, Sichuan University, \\ Chengdu, 610041 Sichuan, P. R. China \\ ${ }^{2}$ Disaster Medical Center, Sichuan \\ University, Chengdu, 610041 Sichuan, \\ P. R. China

\section{*Correspondence \\ yaorong@wchscu.cn \\ (Rong Yao) \\ $\dagger$ These authors contributed equally.}

\begin{abstract}
Objectives: To explore the relationship between alcohol consumption and COVID-19 severity of illness and hospital death.

Methods: This study included 1,087 COVID-19 patients confirmed by laboratory tests in many hospitals in Sichuan Province and Hubei Province during the COVID-19 epidemic. The patients were divided into a drinking group and a nondrinking group. For better baseline feature comparability between the two groups, we used propensity score matching (PSM) to generate a new cohort with a ratio of $1: 2$ (drinking group, $n$ $=167$; nondrinking group, $\mathrm{n}=334$ ) to compare the clinical symptoms, complications, complications, ICU admission and in-hospital death between the drinking group and the nondrinking group and to analyze the factors affecting ICU admission and the prognosis of in-hospital mortality of COVID-19 patients.

Results: The incidence of wheezing symptoms in the nondrinking group was higher than that in the drinking group $(11.2 \%$ vs. $6.6 \%, P=0.044)$ after PSM $(12.3 \%$ vs. $6.6 \%$, $P=0.032$ ), and there was no difference in the incidence of other symptoms between the two groups. The incidence of stroke was lower in the drinking group than in the nondrinking group $(0.0 \%$ vs. $2.4 \%, P=0.025)$ after PSM $(0.0 \%$ vs. $2.7 \%, P=0.025)$. The multivariate analysis showed that drinking was not associated with ICU admission $(\mathrm{OR}=1.240,95 \% \mathrm{CI} 0.322-4.772, P=0.755)$ or in-hospital mortality outcomes $(\mathrm{OR}=$ 2.582, 95\% CI 0.689-9.670, $P=0.159$ ).

Conclusions: Drinking is not associated with patient ICU admission or hospital mortality. However, COVID-19 patients who drink alcohol have fewer wheezing symptoms and fewer stroke complications.
\end{abstract}

\section{Keywords}

Coronavirus disease 2019; Drinking; ICU admission; Wheezing; Stroke; Hospital mortality

\section{Introduction}

Coronavirus disease 2019 (COVID-19) is an infectious disease first reported in Wuhan, Hubei, China, that has spread rapidly around the world $[1,2]$. According to WHO statistics, as of 1 November, nearly 46 million cases and 1.2 million deaths have been reported globally [3]. The main route of transmission of the epidemic is through droplets, aerosols and direct contact interpersonal transmission, with a general incubation period of approximately 2 weeks $[4,5]$. To date, the number of COVID19 cases is increasing rapidly, mainly in the United States, India, Europe and other countries, and the number of deaths is also increasing [6]. COVID-19 occurs at any age but mainly in elderly patients with underlying diseases such as cardiovascular disease, hypertension, diabetes, chronic lung disease and chronic renal disease [7-9]. To date, many countries are working on the development of COVID-19 vaccines to help the global fight against this sudden outbreak.
There are no studies on the correlation between drinking and the prognosis of COVID-19 patients. Only some studies have shown that aggregate alcohol consumption can lead to COVID19 outbreaks that are difficult to control [10]. Related reports note that COVID-19 is more common in elderly patients with basic diseases and that cardiovascular disease, chronic lung disease, and chronic kidney disease are risk factors for COVID-19 critical patients and death [11]. A large number of studies have reported that drinking alcohol has a significant impact on the heart, the brain, the liver, the lungs, blood vessels, immunity and multiple other systems, and this impact may be closely related to the occurrence and development of cardiovascular diseases, hypertension, diabetes, and basic lung diseases [12-15]. Some studies have found that drinking is harmful to the body and a risk factor for critical conditions and death in many basic diseases, but some studies have also indicated that drinking may protect against cardiovascular and cerebrovascular diseases [16, 17]. Therefore, the advantages 
and disadvantages of drinking to the body are controversial.

As a result, we infer that alcohol consumption may have an impact on the prognosis of COVID-19 patients. This study mainly explored the relationship between alcohol consumption and the severity of disease and the prognosis of in-hospital death in patients with COVID-19 infection as well as the factors affecting the severity and prognosis of new coronavirus patients. Our aim was to determine the relationship between alcohol consumption and common diseases in COVID-19 pneumonia patients. We explored the relationship between alcohol consumption and severity of illness and hospital death for COVID-19 to understand the impact of alcohol consumption on disease and to help identify individuals who may progress early, provide better care and medical support, reduce intensive care unit (ICU) stays and improve prognosis.

\section{Materials and methods}

\subsection{Patients}

This study is a retrospective observational study. This study included 1,087 COVID-19 patients confirmed by laboratory tests in many hospitals in Sichuan Province and Hubei Province during the COVID-19 epidemic. The data inclusion deadline of this study was March 14, 2020. All enrolled patients were confirmed to have COVID-19 infection by high quantitative sequencing or real-time reverse transcription polymerase chain reaction (RT-PCR) of nasopharyngeal swab samples and were diagnosed with COVID-19 in accordance with WORLD Health Organization guidelines. The study was approved by the Ethics Committee of West China Hospital of Sichuan University and the Ethics Committee of Wuhan Red Cross Hospital of Hubei Province (2020 (272)).

\subsection{Demographical and risk variables}

Information was collected, including demographic data, comorbidities, history, clinical symptoms, signs at admission, laboratory results at admission, complications, admission therapy measures, and ICU admission. Demographic data included patient age, sex, alcohol use, smoking, time from clinical symptoms until admission, and follow-up until discharge or death. The onset time of clinical symptoms was defined as before the first visit. In this study, the criterion for drinking was drinking more than or equal to three days a week and drinking more than 20 grams per day. The definition of chronic kidney injury (CKD) was based on the 2018 guidelines for chronic kidney function [18]. Categories of chronic liver diseases are reported by Kittichai Promrat [19]. The definition of acute kidney injury (AKI) was based on the 2016 guidelines for acute kidney injury [20]. Acute respiratory distress syndrome (ARDS) was defined according to the report by Eddy Fan [21]. Liver dysfunction was diagnosed when the levels of aminotransferase (AST) and bilirubin were higher than the reference upper limit of the local hospital. Data, including patient diagnosis, were collected from clinical electronic medical records and reviewed, summarized, and crosschecked by a team of experienced clinicians. Each record was examined independently by at least 2 clinicians. For better baseline feature comparability between the two groups, we used propensity score matching (PSM) to generate a new cohort with a ratio of $1: 2$ (drinking group, $\mathrm{n}=167$; nondrinking group, $\mathrm{n}=334$ ). Demographic data, medical history, symptoms, laboratory examination results, ICU hospitalization rate and mortality rate were compared between the two groups, and factors influencing ICU admission and in-hospital death outcome were analyzed.

\subsection{Propensity score matching (PSM) program}

First, all patients were divided into two groups according to whether they drank alcohol: the drinking group and the nondrinking group. We used PSM to increase the comparability between groups. Demographic variables that were not comparable between the two groups were selected $(P>0.10)$ as a covariant factor. Through a logistic regression analysis, these covariate factors were used to calculate the propensity score of each individual in both groups. Then, according to the nearest score, the "nearest" method was used to try to choose 2 matches for each individual in the drinking group from the nondrinking group. These selected patients were eligible for final statistical analysis. This PSM analysis was performed by $\mathrm{R}$ version 3.6.2 using the "MatchIt" package.

\subsection{Statistical analysis}

Categorical variables are expressed as percentages and were compared using a $t$-test. Continuous variables that were normally distributed are represented as the mean \pm standard deviation, and continuous variables that were not normally distributed are represented as the median and quartile intervals and compared using a chi-squared test or Fisher's accuracy test. Risk factors for ICU admission and in-hospital death outcomes were studied using a logistic regression analysis. SPSS 25.0 was used for the statistical analysis, and $P<0.05$ was considered significant.

\section{Results}

\subsection{Clinical characteristics}

A total of 1,087 inpatients with COVID-19 in Sichuan Province and Hubei Province from January 2, 2020 to February 28, 2020 were enrolled. Among them, 920 were nondrinkers with an average age of $51.5 \pm 17.1$ (years), while 167 were drinkers with an average age of $49.5 \pm 17.1$ (years). After PSM, 167 patients were drinkers, and 334 patients were nondrinkers. The proportion of male drinkers (64.7\%) was higher than that of female drinkers (35.3\%), while the drinking group also smoked compared with the nondrinking group (Table 1). The study found that alanine aminotransferase (ALT), aspartate amino transferase (AST), serum creatinine (Cr) and uric acid were significantly higher in the drinking group than in the nondrinking group (Table 2). In the study, the nondrinking group had significantly more wheezing symptoms on admission than the drinking group (11.2\% vs. $6.6 \%, P=0.044)$ after PSM $(12.3 \%$ vs. $6.6 \%, P=0.032)$ (Table 3). Among the underlying complications, the incidence of chronic liver disease was higher in the drinking group than 
TA B L E 1. Baseline characteristics of the included COVID-19 patients with or without alcohol consumption.

\begin{tabular}{|c|c|c|c|c|c|c|c|}
\hline \multirow{2}{*}{\multicolumn{2}{|c|}{ Characteristics }} & \multicolumn{3}{|c|}{ Before PSM } & \multicolumn{3}{|c|}{ After PSM } \\
\hline & & \multicolumn{2}{|c|}{ Nondrinking $(\mathrm{N}=920)$ Drinking $(\mathrm{N}=167)$} & \multirow{2}{*}{$\begin{array}{c}P \\
0.918\end{array}$} & \multirow{2}{*}{$\begin{array}{c}\text { Nondrinking }(\mathrm{N}=334) \\
49.5 \pm 17.1\end{array}$} & \multirow{2}{*}{$\begin{array}{c}\text { Drinking }(\mathrm{N}=167) \\
49.9 \pm 17.7\end{array}$} & \multirow{2}{*}{$\begin{array}{c}P \\
0.392\end{array}$} \\
\hline Age (years) & & $51.5 \pm 17.1$ & $49.5 \pm 17.1$ & & & & \\
\hline Heart rate (beats $/ \mathrm{n}$ & (min) & $87 \pm 14$ & $85 \pm 15$ & 0.585 & $85 \pm 15$ & $87 \pm 13$ & 0.154 \\
\hline RR (breaths/min) & & $20 \pm 3$ & $20 \pm 4$ & 0.730 & $20 \pm 4$ & $20 \pm 3$ & 0.261 \\
\hline $\mathrm{SBP}(\mathrm{mmHg})$ & & $127 \pm 16$ & $127 \pm 17$ & 0.524 & $127 \pm 17$ & $128 \pm 16$ & 0.266 \\
\hline DBP (mmHg) & & $77 \pm 10$ & $78 \pm 12$ & 0.098 & $78 \pm 12$ & $78 \pm 11$ & 0.269 \\
\hline Surplus Pulse $\mathrm{O}_{2}$ & $(\%)$ & $95 \pm 7$ & $95 \pm 5$ & 0.717 & $95 \pm 5$ & $95 \pm 7$ & 0.455 \\
\hline \multicolumn{2}{|l|}{ Sex } & & & $<0.001$ & & & $<0.001$ \\
\hline & Male & $417(45.3 \%)$ & $108(64.7 \%)$ & & $162(48.5 \%)$ & $108(64.7 \%)$ & \\
\hline & Female & $503(54.7 \%)$ & $59(35.3 \%)$ & & $172(51.5 \%)$ & $59(35.3 \%)$ & \\
\hline \multirow[t]{3}{*}{ Smoking } & & & & $<0.001$ & & & $<0.001$ \\
\hline & No & $872(94.8 \%)$ & $52(31.1 \%)$ & & $321(96.1 \%)$ & $52(31.1 \%)$ & \\
\hline & Yes & $48(5.2 \%)$ & $115(68.9 \%)$ & & $13(3.9 \%)$ & $115(68.9 \%)$ & \\
\hline \multirow[t]{3}{*}{ Chronic CVD } & & & & 0.072 & & & 0.159 \\
\hline & Yes & $70(7.60 \%)$ & $7(4.2 \%)$ & & $23(6.9 \%)$ & $7(4.2 \%)$ & \\
\hline & No & $850(92.4 \%)$ & $160(95.8 \%)$ & & $311(93.1 \%)$ & $160(95.8 \%)$ & \\
\hline \multirow[t]{3}{*}{ CPD } & & & & 0.439 & & & 0.474 \\
\hline & Yes & $28(3.0 \%)$ & $4(2.4 \%)$ & & $10(71.4 \%)$ & $4(2.4 \%)$ & \\
\hline & No & $892(97.0 \%)$ & $163(97.6 \%)$ & & $324(28.6 \%)$ & $163(97.6 \%)$ & \\
\hline \multirow[t]{3}{*}{ CKD } & & & & 0.593 & & & 0.624 \\
\hline & Yes & $18(2.0 \%)$ & $3(1.8 \%)$ & & $6(1.8 \%)$ & $3(1.8 \%)$ & \\
\hline & No & $902(98.0 \%)$ & $164(98.2 \%)$ & & $328(98.2 \%)$ & $164(98.2 \%)$ & \\
\hline \multirow[t]{3}{*}{ CLD } & & & & 0.007 & & & 0.008 \\
\hline & Yes & $56(6.1 \%)$ & $20(12.0 \%)$ & & $12(3.6 \%)$ & $20(12.0 \%)$ & \\
\hline & No & $864(3.9 \%)$ & $147(88.0 \%)$ & & $322(96.4 \%)$ & $147(88.0 \%)$ & \\
\hline \multirow[t]{3}{*}{ Cancer } & & & & 0.235 & & & 0.376 \\
\hline & Yes & $23(2.5 \%)$ & $2(1.2 \%)$ & & $7(2.1 \%)$ & $2(1.2 \%)$ & \\
\hline & No & $897(97.5 \%)$ & $165(98.8 \%)$ & & $327(97.9 \%)$ & $165(98.8 \%)$ & \\
\hline \multirow[t]{3}{*}{ Diabetes } & & & & 0.543 & & & 0.436 \\
\hline & Yes & $109(11.8 \%)$ & $20(12.0 \%)$ & & $37(11.1 \%)$ & $20(12.0 \%)$ & \\
\hline & No & $811(88.2 \%)$ & $147(88.0 \%)$ & & $297(88.9 \%)$ & $147(88.0 \%)$ & \\
\hline \multirow[t]{3}{*}{ Hypertension } & & & & 0.267 & & & 0.291 \\
\hline & Yes & $228(24.8 \%)$ & $37(22.2 \%)$ & & $83(24.9 \%)$ & $37(22.2 \%)$ & \\
\hline & No & $692(75.2 \%)$ & $130(77.8 \%)$ & & $251(75.1 \%)$ & $130(77.8 \%)$ & \\
\hline \multirow[t]{3}{*}{ Stroking history } & & & & 0.025 & & & 0.025 \\
\hline & Yes & $22(2.4 \%)$ & $0(0.0 \%)$ & & $9(2.7 \%)$ & $0(0.0 \%)$ & \\
\hline & No & $898(97.6 \%)$ & $167(100.0 \%)$ & & $325(97.3 \%)$ & $167(100.0 \%)$ & \\
\hline
\end{tabular}

$R R$, Respiration rates; SBP, Systolic blood pressure; DBP, Diastolic blood pressure; CVD, cardiovascular disease; $C P D$, Chronic pulmonary diseases; CKD, Chronic kidney diseases; CLD, Chronic liver diseases.

in the nondrinking group $(12.0 \%$ vs. $6.1 \%, P=0.007$; after PSM, $12.0 \%$ vs. $3.6 \%, P=0.008)$, and complications of acute liver dysfunction were also more frequent in the drinking group (18.0\% vs. $9.9 \%, P=0.005$; after PSM $18.0 \%$ vs. $9.1 \%$, $P=0.007)$. However, the incidence of stroke in the drinking group was significantly lower than that in the nondrinking group $(2.4 \%$ vs. $0, \mathrm{P}=0.025$; after PSM, $2.7 \%$ vs. $0, P=$ 0.025 ) (Table 1, Table 4). A total of 97 (8.92\%) patients were admitted to the ICU, and $59(5.42 \%)$ patients died in this study (Tables 4, 5). Among the treatment methods of these patients, a significantly higher proportion of the nondrinking group received glucocorticoid treatment than those in the drinking 
group (208 (22.6\%) vs. 27 (16.2\%), $P=0.037$; after PSM 27 $(16.2 \%)$ vs. $84(25.1 \%), P=0.014)$ (Table 5).

\subsection{Factors associated with ICU hospitalization}

This research adopted a logistic single factor analysis and found that age, smoking status, heart rate, breathing, fever, hypertension, cardiovascular disease, chronic lung disease, chronic kidney disease, hemorrhagic stroke, complications with ARDS, AKL, and LD were risk factors for ICU admission $(P<0.05)$. The multivariate analysis revealed that age $(\geq$ 65 years old vs. $<65$ years old) $(3.686$ (2.239-6.069), $P$ $<0.001)$, respiration $(\geq 22$ times $/ \mathrm{min}$ vs. $<22$ times $/ \mathrm{min})$ (4.016 (2.416-6.676), $P<0.001)$, clinical symptoms of dyspnea $(1.729(1.014-2.950), P=0.044)$, smoking status $(2.032$ (1.114-3.706), $P=0.021)$, CKD (10.686 (3.243-35.214), $P$ $<0.001)$, ARDS (10.868 (5.271-22.409), $P<0.001)$, and AKI (3.348 (1.051-10.660), $P=0.041)$ were independent risk factors for ICU hospitalization (Fig. 1).

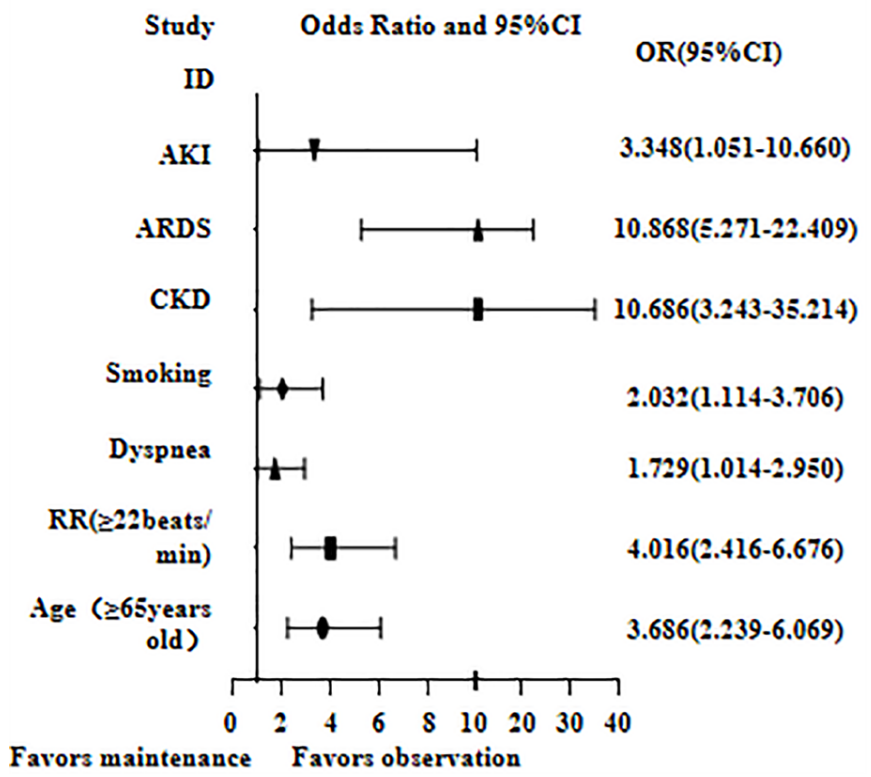

F I G URE 1. Logistic regression analysis of risk factors associated with ICU hospitalization.

\subsection{Factors associated with patient in-hospital death}

The logistic univariate analysis found that sex, age, vital signs, heart rate and respiration at admission, complications with hypertension, cardiovascular disease, chronic lung disease, chronic kidney disease, history of stroke, blood system disease, and ARDS were risk factors for in-hospital death in patients $(P<0.05)$. The multivariate analysis revealed that age $(\geq 65$ years vs. $<65$ years) $(10.954$ (4.955-24.214), $P<0.001)$, heart rate $(\geq 100$ beats $/ \mathrm{min}$ vs. $<$ 100 times/min), 2.290 (1.016 5.162), $P=0.046)$, respiratory (22 times per minute or higher vs. $<22$ times/min $(2.888$ (1.322-6.308)), $P<0.008)$, complications with hematological diseases ((59.283(1.567-2242.643), $P=0.028)$, CKD (10.538 (2.720-40.830), $P=0.001)$, and ARDS (3.394 (1.266-9.093),
$P=0.015)$, TCM $(0.381(0.183-0.790), P=0.010)$ were independent risk factors for in-hospital death (Fig. 2).

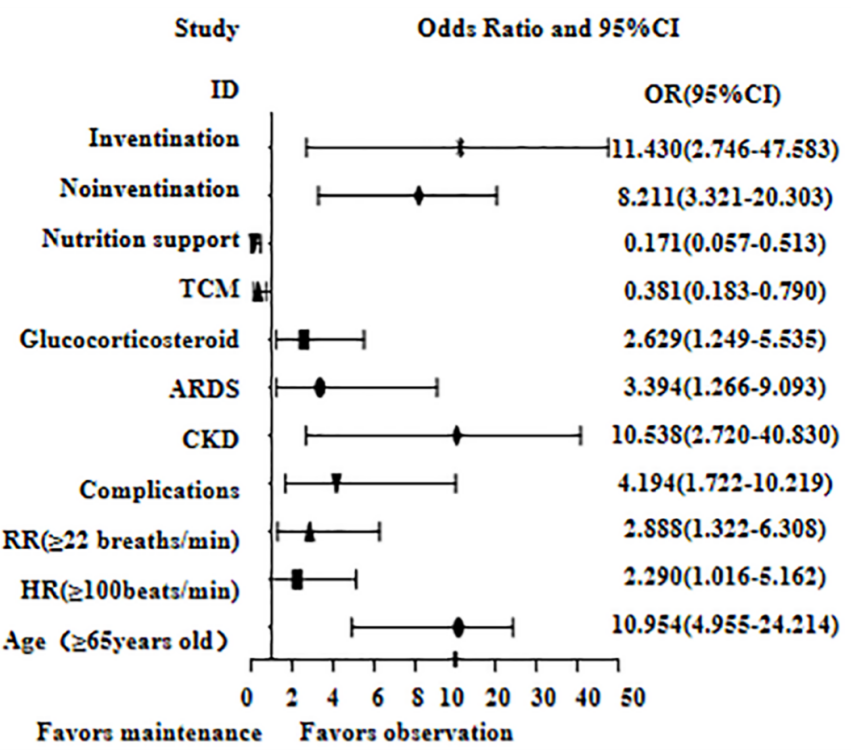

FIGURE 2. Logistic regression analysis of risk factors associated with in-hospital mortality.

\section{Discussion}

COVID-19 is a global battle; as a disease that mainly invades the lungs, it highly affects patients with underlying chronic lung disease, cardiovascular disease, renal function disease, hypertension, and diabetes and the elderly [4, 22-24]. However, our living and eating habits are closely related to the occurrence of these lesions. Among them, drinking is a very common eating habit. Whether in China or abroad, alcohol has a long-term and complex role in human health, and excessive drinking causes great morbidity and mortality [25]. Previous studies have found that alcohol affects many organs, including the heart, brain, lungs, liver and kidneys; blood vessels; and the immune system. The advantages and disadvantages of alcohol to the body mainly lie in the content and timing of alcohol intake. At present, most of the diseases reported related to alcohol effects include hepatitis cirrhosis, pancreatitis, coronary heart disease, nervous system disease, and psychosis [26-28]. At the same time, alcohol affects the immune system and interferes with the immune response, including cell-mediated and humoral responses [29]. Therefore, some studies suggest that alcohol drinkers are more likely to develop pneumonia, tuberculosis, acute respiratory distress syndrome and other pneumonia diseases [30]. However, some studies also show that light and moderate drinking has a protective effect on the cardiovascular system compared with long-term heavy drinking [16]. Therefore, this study mainly explored the relationship between alcohol consumption and the severity of COVID-19 infection and the prognosis of death as well as the factors affecting the severity and prognosis of new coronavirus patients. This study explored a group analysis according to the drinking behaviors of patients.

Our study found that the rate of wheezing symptoms in the drinking group was lower than that in the nondrinking 
TA B L E 2. Lab findings for COVID-19 patients: drinking and non-drinking groups.

\begin{tabular}{|c|c|c|c|c|c|c|}
\hline \multirow[t]{2}{*}{ Varibles } & \multicolumn{3}{|c|}{ Before PSM } & \multicolumn{3}{|c|}{ After PSM } \\
\hline & Nondrinking $(\mathrm{N}=920)$ & Drinking $(\mathrm{N}=167)$ & $P$ & Nondrinking $(\mathrm{N}=334)$ & Drinking $(\mathrm{N}=167)$ & $P$ \\
\hline $\operatorname{WBC}\left(10^{9} / \mathrm{L}\right)$ & $6.14 \pm 3.49$ & $5.89 \pm 2.30$ & 0.313 & $6.48 \pm 4.20$ & $5.89 \pm 2.30$ & 0.078 \\
\hline $\mathrm{Hb}(\mathrm{g} / \mathrm{L})$ & $127 \pm 22$ & $138 \pm 28$ & 0.178 & $129 \pm 20$ & $138 \pm 28$ & 0.052 \\
\hline HCT & $0.38(0.35,0.41)$ & $0.41(0.36,0.45)$ & $<0.001$ & $0.38(0.35,0.41)$ & $0.41(0.36,0.45)$ & 0.003 \\
\hline Platelets $\left(10^{9} / \mathrm{L}\right)$ & $218 \pm 94$ & $209 \pm 82$ & 0.069 & $218 \pm 88$ & $209 \pm 82$ & 0.123 \\
\hline D-dimer & $0.56(0.27,1.41)$ & $0.60(0.33,1.64)$ & 0.283 & $0.58(0.30,1.79)$ & $0.60(0.33,1.64)$ & 0.769 \\
\hline FIB $(g / L)$ & $3.64(2.60,4.82)$ & $4.19(3.19,5.47)$ & 0.006 & $3.58(2.67,4.68)$ & $4.19(3.19,5.47)$ & 0.008 \\
\hline APTT (S) & $28.0(25.9,31.6)$ & $29.6(27.1,33.6)$ & 0.003 & $27.9(25.2,31.6)$ & $29.6(27.1,33.6)$ & 0.003 \\
\hline $\mathrm{PT}(\mathrm{S})$ & $12.1(11.4,12.9)$ & $12.0(11.3,13.1)$ & 0.865 & $12.0(11.2,12.8)$ & $12.0(11.3,13.1)$ & 0.496 \\
\hline INR & $1.05 \pm 0.12$ & $1.05 \pm 0.12$ & 0.476 & $1.04 \pm 0.11$ & $1.05 \pm 0.12$ & 0.467 \\
\hline TBIL $(\mu \mathrm{mol} / \mathrm{L})$ & $9.9(7.3,14.0)$ & $10.5(7.3,15.2)$ & 0.36 & $10.1(7.3,14.0)$ & $10.5(7.3,15.2)$ & 0.582 \\
\hline DBIL $(\mu \mathrm{mol} / \mathrm{L})$ & $3.3(2.3,4.6)$ & $3.6(2.4,5.4)$ & 0.044 & $3.1(2.2,4.5)$ & $3.6(2.4,5.4)$ & 0.031 \\
\hline ALT (U/L) & $22.0(15.1,37.0)$ & $31.0(18.0,54.2)$ & $<0.001$ & $22.0(15.9,36.3)$ & $31.0(18.0$ & $<0.001$ \\
\hline AST (U/L) & $24.0(19.0,34.1)$ & $29.0(21.5,42.0)$ & 0.003 & $24.0(20.0,33.0)$ & $29.0(21.5,42.0)$ & 0.001 \\
\hline $\operatorname{ALB}(g / L)$ & $39.11 \pm 5.98$ & $40.89 \pm 5.69$ & 0.809 & $39.07 \pm 6.52$ & $40.89 \pm 5.69$ & 0.869 \\
\hline TG mmol/L & $1.53 \pm 0.99$ & $1.55 \pm 0.96$ & 0.644 & $1.61 \pm 1.12$ & $1.55 \pm 0$ & 0.222 \\
\hline $\mathrm{CHOL} \mathrm{mmol} / \mathrm{L}$ & $3.94(3.41,4.58)$ & $3.98(3.33,4.30)$ & 0.393 & $4.04(3.49,4.79)$ & $3.98(3.33,4.30)$ & 0.137 \\
\hline $\mathrm{HDL}-\mathrm{C} \mathrm{mmol} / \mathrm{L}$ & $1.10 \pm 0.47$ & $1.03 \pm 0.31$ & 0.435 & $1.15 \pm 0.61$ & $1.03 \pm 0.31$ & 0.080 \\
\hline LDL-C mmol/L & $2.41 \pm 0.76$ & $2.33 \pm 0.70$ & 0.581 & $2.43 \pm 0.71$ & $2.33 \pm$ & 0.769 \\
\hline CK-MB (U/L) & $1.88(0.80,11.00)$ & $7.5(1.12,12.68)$ & 0.011 & $2.56(0.89,11.73)$ & $7.5(1.12,12.68)$ & 0.292 \\
\hline CK (U/L) & $60(39,101)$ & $74(44,129)$ & 0.456 & $57(35,105)$ & $74(44,129)$ & 0.033 \\
\hline Glocose $(\mathrm{mmol} / \mathrm{L})$ & $5.67(4.91,7.06)$ & $5.84(5.10,6.97)$ & 0.327 & $5.77(4.91,7.13)$ & $5.84(5.10,6.97)$ & 0.585 \\
\hline BUN (mmol/L) & $4.10(3.30,5.50)$ & $4.48(3.49,5.60)$ & 0.143 & $4.00(3.25,5.40)$ & $4.48(3.49,5.60)$ & 0.111 \\
\hline $\mathrm{Cr}(\mu \mathrm{mol} / \mathrm{L})$ & $261(199,331)$ & $69(55,79)$ & $<0.001$ & $61(51,73)$ & $69(55,79)$ & 0.002 \\
\hline $\mathrm{eGFR}(\mathrm{ml} / \mathrm{min} / 1.73 \mathrm{~m})$ & $99.31 \pm 25.4$ & $97.45 \pm 25.9$ & 0.974 & $101.92 \pm 28.99$ & $97.45 \pm 25.9$ & 0.663 \\
\hline Uric acid $(\mu \mathrm{mol} / \mathrm{L})$ & $261(199,331)$ & $292(222,363)$ & 0.002 & $265(198,324)$ & $292(222,363)$ & 0.004 \\
\hline TNT-I (ng/mL) & $0.02(0.01,0.10)$ & $0.02(0.01,0.04)$ & 0.395 & $0.02(0.01,0.10)$ & $0.02(0.01,0.04)$ & 0.553 \\
\hline pro-BNP (pg/mL) & $77.8(31.8,259.4)$ & $114.2(9.3,236.2)$ & 0.862 & $94.6(35.8,245.6)$ & $114.2(9.3,236.2)$ & 0.849 \\
\hline $\begin{array}{l}\mathrm{CD} 3 \text { cell count } \\
(\mathrm{cell} / \mathrm{uL})\end{array}$ & $775(457,1052)$ & $743(512,1138)$ & 0.833 & $795(451,1073)$ & $743(512,1138)$ & 0.945 \\
\hline $\begin{array}{l}\mathrm{CD} 4 \text { cell count } \\
(\mathrm{cell} / \mathrm{uL})\end{array}$ & $456(279,671)$ & $456(341,662)$ & 0.610 & $456(250,666)$ & $456(341,662)$ & 0.457 \\
\hline $\begin{array}{l}\text { CD8 cell count } \\
(\text { cell } / \mathrm{uL})\end{array}$ & 262 & 86) & 0.523 & 85) & 232 & 0.601 \\
\hline C3 (C3) (g/L) & $1.05 \pm 0.21$ & $1.16 \pm 0.17$ & 0.195 & $1.09 \pm 0.21$ & $1.16 \pm 0.17$ & 0.059 \\
\hline C4 (C4) (g/L) & $0.27 \pm 0.10$ & $0.30 \pm 0.07$ & 0.033 & $0.28 \pm 0.10$ & $0.30 \pm 0.07$ & 0.217 \\
\hline C-Protein $(\mathrm{mg} / \mathrm{L})$ & $20.4(7.2,57.9)$ & $26.4(10.2,45.2)$ & 0.472 & $19.7(7.4,56.7)$ & $26.4(10.2,45.2)$ & 0.307 \\
\hline
\end{tabular}

WBC, White blood cell count; Hb, Hemoglobin; HCT, Hematocrit; FIB, Fibrinogen; APTT, Activated partial thromboplastin time; PT, Prothrombin time; INR, International normalized ratio; TBIL, Total bilirubin; DBIL, Direct bilirubin; ALT, Alanine aminotransferase; AST, Aspartate amino transferase; ALB, Albumin; CHOL, Cholesterol; TG, Triglyceride; HDL-C, High-density lipoprotein cholesterol; LDL-C, Low-density lipoprotein cholesterol; CK, Creatine kinase; CK-MB, Creatine kinase isoenzyme; $B U N$, urea nitrogen; $C r$, Serum creatinine; eGFR, glomerular filtration rate;TNT-I, Hypersensitive Troponin-I.

group, and the utilization rate of glucocorticoids in the drinking group was lower than that in the nondrinking group, which was consistent with the use of glucocorticoid drugs when necessary in our clinical treatment. The effect of drinking on pulmonary airway function depends on alcohol concentration and alcohol duration. The low incidence of wheezing symptoms in drinking groups may be due to transient exposure to low concentrations of alcohol which increases steroid hormone levels, 
TA B L E 3. Symptoms between COVID-19 patients in the drinking and nondrinking groups.

\begin{tabular}{|c|c|c|c|c|c|c|c|}
\hline \multirow[t]{2}{*}{ Symptoms } & & \multicolumn{3}{|c|}{ Before PSM } & \multicolumn{3}{|c|}{ After PSM } \\
\hline & & Nondrinking & Drinking & $P$ & Nondrinking & Drinking & $P$ \\
\hline \multirow[t]{2}{*}{ Fever } & Yes & $583(63.4 \%)$ & $112(67.1 \%)$ & 0.204 & $212(63.5 \%)$ & $112(67.1 \%)$ & 0.244 \\
\hline & No & $337(36.6 \%)$ & $55(36.1 \%)$ & & $122(36.5 \%)$ & $55(36.1 \%)$ & \\
\hline \multirow[t]{2}{*}{ Dry cough } & Yes & $560(60.9 \%)$ & $107(61.4 \%)$ & 0.244 & $211(63.2 \%)$ & $107(61.4 \%)$ & 0.462 \\
\hline & No & $360(39.1 \%)$ & $60(35.9 \%)$ & & $123(36.8 \%)$ & $60(35.9 \%)$ & \\
\hline \multirow[t]{2}{*}{ Expectoration } & Yes & $276(30.0 \%)$ & $59(35.3 \%)$ & 0.101 & $276(30.0 \%)$ & $59(35.3 \%)$ & 0.101 \\
\hline & No & $644(70.0 \%)$ & $108(64.7 \%)$ & & $644(70.0 \%)$ & $108(64.7 \%)$ & \\
\hline \multirow[t]{2}{*}{ Dyspnea } & Yes & $182(19.8 \%)$ & $33(19.8 \%)$ & 0.545 & $81(24.3 \%)$ & $33(19.8 \%)$ & 0.154 \\
\hline & No & $738(80.2 \%)$ & $134(80.2 \%)$ & & $253(75.7 \%)$ & $134(80.2 \%)$ & \\
\hline \multirow[t]{2}{*}{ Weakness } & Yes & $346(37.6 \%)$ & $61(36.5 \%)$ & 0.431 & $138(41.3 \%)$ & $61(36.5 \%)$ & 0.175 \\
\hline & No & $574(62.4 \%)$ & $106(63.5 \%)$ & & $196(58.7 \%)$ & $106(63.5 \%)$ & \\
\hline \multirow[t]{2}{*}{ Sore throat } & Yes & $95(10.3 \%)$ & $16(9.6 \%)$ & 0.449 & $39(11.7 \%)$ & $16(9.6 \%)$ & 0.293 \\
\hline & No & $825(89.7 \%)$ & $151(90.4 \%)$ & & $295(88.3 \%)$ & $151(90.4 \%)$ & \\
\hline \multirow[t]{2}{*}{ Nasal congestion } & Yes & $37(4.0 \%)$ & $5(3.0 \%)$ & 0.354 & $14(4.2 \%)$ & $5(3.0 \%)$ & 0.348 \\
\hline & No & $883(96.0 \%)$ & $162(97.0 \%)$ & & $320(95.8 \%)$ & $162(97.0 \%)$ & \\
\hline \multirow[t]{2}{*}{ Wheezing } & Yes & $103(11.2 \%)$ & $11(6.6 \%)$ & 0.044 & $41(12.3 \%)$ & $11(6.6 \%)$ & 0.032 \\
\hline & No & $817(88.8 \%)$ & $156(3.4 \%)$ & & $293(87.7 \%)$ & $156(3.4 \%)$ & \\
\hline \multirow[t]{2}{*}{ Chest discomfort } & Yes & $172(18.7 \%)$ & $30(18.0 \%)$ & 0.46 & $75(22.5 \%)$ & $30(18.0 \%)$ & 0.147 \\
\hline & No & $748(81.3 \%)$ & $137(82.0 \%)$ & & $259(77.5 \%)$ & $137(82.0 \%)$ & \\
\hline \multirow[t]{2}{*}{ Arthrodynia } & Yes & $93(10.1 \%)$ & $20(12.0 \%)$ & 0.272 & $30(9.0 \%)$ & $20(12.0 \%)$ & 0.184 \\
\hline & No & $827(89.9 \%)$ & $107(89.2 \%)$ & & $334(91.0 \%)$ & $107(89.2 \%)$ & \\
\hline \multirow[t]{2}{*}{ Nausea and vomiting } & Yes & $36(3.9 \%)$ & $5(3.0 \%)$ & 0.379 & $16(4.8 \%)$ & $5(3.0 \%)$ & 0.243 \\
\hline & No & $884(96.1 \%)$ & $162(97.0 \%)$ & & $318(95.2 \%)$ & $162(97.0 \%)$ & \\
\hline \multirow[t]{2}{*}{ Diarrhea } & Yes & $92(10.0 \%)$ & $18(10.8 \%)$ & 0.424 & $32(9.6 \%)$ & $18(10.8 \%)$ & 0.391 \\
\hline & No & $828(90.0 \%)$ & $149(89.2 \%)$ & & $302(90.4 \%)$ & $149(89.2 \%)$ & \\
\hline
\end{tabular}

TA B L E 4. Complications between COVID-19 patients in drinking and nondrinking groups.

\begin{tabular}{|c|c|c|c|c|c|c|c|}
\hline & & & fore PSM & & & After PSM & \\
\hline Complications & & Drinking & Nondrinking & $P$ & Nondrinking & Drinking & $P$ \\
\hline Total Complications & Yes & $80(47.9 \%)$ & $407(44.2 \%)$ & 0.214 & $137(41.0 \%)$ & $80(47.9 \%)$ & 0.085 \\
\hline & No & $87(52.1 \%)$ & $513(55.8 \%)$ & & $197(59.0 \%)$ & $87(52.1 \%)$ & \\
\hline Bacteria pneumonia & Yes & $18(10.8 \%)$ & $118(13.1 \%)$ & 0.443 & $49(14.9 \%)$ & $18(10.8 \%)$ & 0.195 \\
\hline & No & $149(89.2 \%)$ & $786(86.9 \%)$ & & $279(85.1 \%)$ & $149(89.2 \%)$ & \\
\hline ARDS & Yes & $7(4.2 \%)$ & $39(4.4 \%)$ & 0.410 & $18(5.5 \%)$ & $7(4.2 \%)$ & 0.410 \\
\hline & No & $160(95.8 \%)$ & $856(95.6 \%)$ & & $307(94.5 \%)$ & $160(95.8 \%)$ & \\
\hline Hydrothorax & Yes & $5(3.0 \%)$ & $30(3.3 \%)$ & 0.92 & $12(3.7 \%)$ & $5(3.0 \%)$ & 0.907 \\
\hline & No & $162(97.0 \%)$ & $873(96.7 \%)$ & & $316(96.3 \%)$ & $162(97.0 \%)$ & \\
\hline AKI & Yes & $2(1.2 \%)$ & $14(1.6 \%)$ & 0.489 & $4(1.2 \%)$ & $2(1.2 \%)$ & 0.358 \\
\hline & No & $165(98.8 \%)$ & $889(98.4 \%)$ & & $324(98.8 \%)$ & $165(98.8 \%)$ & \\
\hline Liver dysfunction & Yes & $30(18.0 \%)$ & $89(9.9 \%)$ & 0.005 & $30(9.1 \%)$ & $30(18.0 \%)$ & 0.007 \\
\hline & No & $137(82 \%)$ & $807(90.1 \%)$ & & $298(90.9 \%)$ & $137(82 \%)$ & \\
\hline Death & Yes & $7(4.3 \%)$ & $52(5.7 \%)$ & 0.29 & $16(4.8 \%)$ & $7(4.3 \%)$ & 0.479 \\
\hline & No & $154(95.6 \%)$ & $868(94.3 \%)$ & & $334(95.2 \%)$ & $154(95.6 \%)$ & \\
\hline
\end{tabular}

ARDS, Acute respiratory distress syndrome; AKI, Acute kidney injury. 
TA B L E 5. Treatments between COVID-19 patients in drinking and nondrinking groups.

\begin{tabular}{|c|c|c|c|c|c|c|c|}
\hline \multirow{2}{*}{ Treatments } & & \multicolumn{3}{|c|}{ Before PSM } & \multicolumn{3}{|c|}{ After PSM } \\
\hline & & Drinking & Nondrinking & $P$ & Drinking & Nondrinking & $P$ \\
\hline \multirow[t]{2}{*}{ ICU admission } & Yes & $18(10.8 \%)$ & $79(8.6 \%)$ & 0.218 & $18(10.8 \%)$ & $28(8.4 \%)$ & 0.236 \\
\hline & No & $149(90.2 \%)$ & $841(91.4 \%)$ & & $149(90.2 \%)$ & $306(91.6 \%)$ & \\
\hline \multirow[t]{2}{*}{ Noninvasive ventilation } & Yes & $8(4.8 \%)$ & $65(7.1 \%)$ & 0.182 & $8(4.8 \%)$ & $26(7.8 \%)$ & 0.142 \\
\hline & No & $159(95.2 \%)$ & $855(92.9 \%)$ & & $159(95.2 \%)$ & $308(92.2 \%)$ & \\
\hline \multirow[t]{2}{*}{ Invasive ventilation } & Yes & $2(1.2 \%)$ & $16(1.7 \%)$ & 0.461 & $2(1.2 \%)$ & $7(2.1 \%)$ & 0.376 \\
\hline & No & $165(98.8 \%)$ & $904(98.3 \%)$ & & $165(98.8 \%)$ & $327(97.9 \%)$ & \\
\hline \multirow[t]{2}{*}{ Antiviral drugs } & Yes & $150(89.8 \%)$ & $874(95.0 \%)$ & 0.01 & $150(89.8 \%)$ & $16(48.5 \%)$ & 0.020 \\
\hline & No & $17(10.2 \%)$ & $46(5.0 \%)$ & & $17(10.2 \%)$ & $46(51.5 \%)$ & \\
\hline \multirow[t]{2}{*}{ Antibiotics } & Yes & $88(52.7 \%)$ & $539(58.6 \%)$ & 0.092 & $88(52.7 \%)$ & $201(60.2 \%)$ & 0.067 \\
\hline & No & $79(47.3 \%)$ & $381(41.4 \%)$ & & $79(47.3 \%)$ & $133(39.8 \%)$ & \\
\hline \multirow[t]{2}{*}{ Corticosteroid } & Yes & $27(16.2 \%)$ & $208(22.6 \%)$ & 0.037 & $27(16.2 \%)$ & $84(25.1 \%)$ & 0.014 \\
\hline & No & $140(83.8 \%)$ & $712(77.4 \%)$ & & $140(83.8 \%)$ & $250(74.1 \%)$ & \\
\hline \multirow[t]{2}{*}{ Nutritional support } & Yes & $10(6.0 \%)$ & $107(11.6 \%)$ & 0.017 & $10(6.0 \%)$ & $40(12.0 \%)$ & 0.023 \\
\hline & No & $157(94.0 \%)$ & $813(88.4 \%)$ & & $157(94.0 \%)$ & $294(88.0 \%)$ & \\
\hline \multirow[t]{2}{*}{$\mathrm{TCM}$} & Yes & $139(83.2 \%)$ & $690(75.0 \%)$ & 0.012 & $139(83.2 \%)$ & $245(73.4 \%)$ & 0.008 \\
\hline & No & $28(16.7 \%)$ & $230(25.0 \%)$ & & $28(16.7 \%)$ & $89(26.6 \%)$ & \\
\hline
\end{tabular}

ICU, intensive care unit; TCM, traditional Chinese medicine.

reduces some inflammatory molecules such as IL-6 and CRP, enhances mucosal cilia clearance, stimulates bronchiectasis, and may reduce airway inflammation and injury observed in asthma and chronic obstructive pulmonary disease (COPD) [31]. Similarly, some studies suggest that moderate shortterm drinking can improve respiratory symptoms [31, 32]. However, heavy drinking may play the opposite role, and more studies have shown that long-term heavy drinking makes the host vulnerable to lung diseases such as pneumonia, tuberculosis, and acute respiratory distress syndrome [33]. This is mainly associated with excessive alcohol consumption leading to the formation of reactive aldehydes in the lung, which react with nucleophilic targets in cells to form compounds that may interfere with cell function, disrupt proteins, nucleic acids, etc. This causes abnormal synthesis and secretion of pulmonary surfactants as well as increased apoptosis in II cells and play a role in the pathobiology of airway mucus, bronchial blood flow, and airway smooth muscle regulation [30]. Alcohol exposure time, alcohol consumption concentration and alcohol consumption of COVID-19 patients were not measured in this study, so the advantages and disadvantages of drinking for lung diseases still need to be further discussed.

Our study showed that the incidence of stroke in the drinking group of COVID-19 patients was lower than that in the nondrinking group and negatively correlated with the incidence and mortality of stroke during moderate drinking in some studies, and the conclusion that moderate drinking was a protective factor for stroke was consistent [17]. The main reason for this finding may be that a low alcohol concentration increases high-density lipoprotein cholesterol levels, reduces platelet aggregation, increases fibrinolysis, reduces plasma fibrinogen levels, and achieves antithrombotic activity [17, 34]. However, most of the results are the same as those of some ischemic stroke studies, and there is still controversy about hemorrhagic stroke, which is likely to be related to hypertension caused by drinking. At the same time, long-term heavy drinking increases the risk of stroke. The association between alcohol intake and stroke morbidity and mortality was U shaped [34]. This study did not identify the type of stroke, so it failed to exclude the interference of ischemic and hemorrhagic diseases.

Our study found that drinking is not an independent risk factor for the prognosis of ICU admission and in-hospital death. There was no significant difference in ICU admission or in-hospital mortality between the drinking group and the nondrinking group. Age ( $>65$ years), respiratory rate (22 breaths/min), underlying chronic kidney disease and ARDS are associated with ICU admission and hospital mortality, which is consistent with most previous studies [35]. Previous research has identified that comorbidities, especially cardiovascular system disease, play a key role in the prognosis of patients with COVID-19 $[8,36]$. In this study, we observed that patients with CKD and AKI were more likely to be admitted to the ICU and that kidney disease was an independent risk factor for ICU admission in patients with COVID-19. This finding suggested that patients with a comorbidity of CKD on admission possibly had a high risk of deterioration. This study also found that the incidence of chronic liver disease in the drinking group was significantly higher than that in the nondrinking group, and the complication rate of liver function injury in the drinking group was significantly higher than that in the nondrinking group. This is in line with most previous studies indicating that alcohol intake can lead to elevated serum aminotransferase, liver inflammatory response, and oxidative/nitrification stress and then induce hepatic steatosis and metabolic disorders [37, 
38].

The limitations of this study are as follows: first, this study is a retrospective observational study; second, the alcohol consumption in this study did not specify the patient's alcohol exposure time, alcohol concentration, alcohol category or other detailed information; third, stroke subtypes (hemorrhagic stroke and ischemic stroke) were not classified in this study because they may have different effects on alcohol exposure; fourth, this study is a multicenter study mainly comprising the clinical databases of hospitals in Sichuan Province and Wuhan, Hubei Province. The timing and dosage of drugs used in patient treatment, which may affect the prognosis of patients, were not provided in detail.

\section{Conclusions}

Drinking is not associated with the patient's ICU admission or hospital mortality. However, COVID-19 patients who drink alcohol had fewer wheezing symptoms and fewer stroke complications.

\section{ABBREVIATIONS}

AKI, acute renal insufficiency; ARDS, acute respiratory distress syndrome; CI, confidence interval; CKD, chronic kidney disease; CLD, chronic liver disease; COPD, chronic obstructive pulmonary disease; COVID-19, coronavirus disease 2019; CRD, chronic respiratory disease; CVD, cardiovascular disease; ICU, intensive care unit; OR, odds ratio; TCM, traditional Chinese medicine.

\section{AUTHOR CONTRIBUTIONS}

Conception and design: Lin Lv, Yiwu Zhou, Yao Rong. Collection and assembly of data: Lin Lv, Yiwu Zhou. Data analyses and interpretation: Lin Lv, Yiwu Zhou, Yao Rong. Manuscript preparation: Lin Lv, Yiwu Zhou. Manuscript proofing: all authors. Final approval of the manuscript: all authors.

\section{ETHICS APPROVAL AND CONSENT TO PARTICIPATE}

The study was approved by the Ethics Committee of West China Hospital of Sichuan University and the Ethics Committee of Wuhan Red Cross Hospital of Hubei Province (2020 (272)).

After institutional review board approval was provided at each institution, written informed consent was obtained from each patient or the patient's legally authorized surrogate before conducting study-specific procedures.

\section{ACKNOWLEDGMENT}

I would like to express my gratitude to all those who helped me during the writing of this manuscript.

\section{FUNDING}

This study was supported by the Emergency Response Project for New Coronavirus of Science and Technology Department of Sichuan Province (No. 2020YFS0009, 2020YFS0005), the Special Funds for COVID-19 Prevention and Control of West China Hospital of Sichuan University (No. HX-2019nCoV-068) and the Special Funds for COVID-19 Prevention and Control of Chengdu Science and Technology Bureau (No. 2020-YF05-00074-SN).

\section{CONFLICT OF INTEREST}

The authors have no conflicts of interest to declare.

\section{AVAILABILITY OF DATA AND MATERIALS}

The datasets used and/or analyzed in the present study are available from the corresponding author upon reasonable request.

\section{REFERENCES}

[1] Zhou P, Yang X, Wang X, Hu B, Zhang L, Zhang W, et al. A pneumonia outbreak associated with a new coronavirus of probable bat origin. Nature. 2020; 579: 270-273.

[2] Hui DS, I Azhar E, Madani TA, Ntoumi F, Kock R, Dar O, et al. The continuing 2019-nCoV epidemic threat of novel coronaviruses to global health - the latest 2019 novel coronavirus outbreak in Wuhan, China. International Journal of Infectious Diseases. 2020; 91: 264-266.

[3] World Health Organization. Weekly epidemiological update - 3 November 2020. 2020. Available at: https://www.who.int/ publications/m/item/weekly-epidemiological-update--3-november-2020.

[4] Zhou Y, He Y, Yang H, Yu H, Wang T, Chen Z, et al. Exploiting an early warning Nomogram for predicting the risk of ICU admission in patients with COVID-19: a multi-center study in China. Scandinavian Journal of Trauma, Resuscitation and Emergency Medicine. 2020; 28: 106.

[5] Machhi J, Herskovitz J, Senan AM, Dutta D, Nath B, Oleynikov MD, et al. The natural history, pathobiology, and clinical manifestations of SARS-CoV-2 infections. Journal of Neuroimmune Pharmacology. 2020; 15: $359-386$

[6] Van Damme W, Dahake R, Delamou A, Ingelbeen B, Wouters E, Vanham $\mathrm{G}$, et al. The COVID-19 pandemic: diverse contexts; different epidemicshow and why? BMJ Global Health. 2020; 5: e003098.

[7] Rajapakse N, Dixit D. Human and novel coronavirus infections in children: a review. Paediatrics and International Child Health. 2020; 120.

[8] Nishiga M, Wang DW, Han Y, Lewis DB, Wu JC. COVID-19 and cardiovascular disease: from basic mechanisms to clinical perspectives. Nature Reviews Cardiology. 2020; 17: 543-558.

[9] Sockrider M, Tal-Singer R. Managing your chronic lung disease during the COVID-19 pandemic. American Journal of Respiratory and Critical Care Medicine. 2020; 202: P5-P6.

[10] Chick J. Alcohol and COVID-19. Alcohol and Alcoholism. 2020; 55: 341-342.

[11] Angel-Korman A, Brosh T, Glick K, Leiba A. Covid-19, the kidney and hypertension. Harefuah. 2020; 159: 231-234. (In Hebrew)

[12] Harris B, McAlister A, Willoughby T, Sivaraman V. Alcohol-dependent pulmonary inflammation: a role for HMGB-1. Alcohol. 2019; 80: 45-52.

[13] Szabo G, Saha B. Alcohol's effect on host defense. Alcohol Research: Current Reviews. 2015; 37: 159-170.

[14] Rehm J, Roerecke M. Cardiovascular effects of alcohol consumption. Trends in Cardiovascular Medicine. 2017; 27: 534-538. 
[15] Campollo O. Alcohol and the liver: the return of the prodigal son. Annals of Hepatology. 2019; 18: 6-10.

[16] Krenz M, Korthuis RJ. Moderate ethanol ingestion and cardiovascular protection: from epidemiologic associations to cellular mechanisms. Journal of Molecular and Cellular Cardiology. 2012; 52: 93-104.

[17] Zhang C, Qin Y, Chen Q, Jiang H, Chen X, Xu C, et al. Alcohol intake and risk of stroke: a dose-response meta-analysis of prospective studies. International Journal of Cardiology. 2014; 174: 669-677.

[18] Japanese Society of Nephrology. Essential points from evidence-based clinical practice guidelines for chronic kidney disease 2018. Clinical and Experimental Nephrology. 2019; 23: 1-15.

[19] Chung W, Promrat K, Wands J. Clinical implications, diagnosis, and management of diabetes in patients with chronic liver diseases. World Journal of Hepatology. 2020; 12: 533-557.

[20] Doi K, Nishida O, Shigematsu T, Sadahiro T, Itami N, Iseki K, et al. The Japanese clinical practice guideline for acute kidney injury 2016. Clinical and Experimental Nephrology. 2018; 22: 985-1045.

[21] Fan E, Brodie D, Slutsky AS. Acute respiratory distress syndrome: advances in diagnosis and treatment. Journal of the American Medical Association. 2018; 319: 698.

[22] Vellas C, Delobel P, De Souto Barreto P, Izopet J. COVID-19, virology and geroscience: a perspective. The Journal of Nutrition, Health \& Aging. 2020; 24: 685-691.

[23] Li H, Liu SM, Yu XH, Tang SL, Tang CK. Coronavirus disease 2019 (COVID-19): current status and future perspectives. International Journal of Antimicrobial Agents. 2020; 55: 105951.

[24] Wang D, Hu B, Hu C, Zhu F, Liu X, Zhang J, et al. Clinical characteristics of 138 hospitalized patients with 2019 novel coronavirusinfected pneumonia in Wuhan, China. Journal of the American Medical Association. 2020; 323: 1061

[25] Gonzales K, Roeber J, Kanny D, Tran A, Saiki C, Johnson H, et al. Alcohol-attributable deaths and years of potential life lost-11 States, 2006-2010. Morbidity and Mortality Weekly Report. 2014; 63: 213-216.

[26] Goel S, Sharma A, Garg A. Effect of Alcohol Consumption on Cardiovascular Health. Current Cardiology Reports. 2018; 20: 19.

[27] Matsushita S, Higuchi S. Alcohol and the risk of dementia. Brain and Nerves. 2016; 68: 819-827. (In Japanese)

[28] Kodali S, Kaif M, Tariq R, Singal AK. Alcohol relapse after liver transplantation for alcoholic cirrhosis-impact on liver graft and patient survival: a meta-analysis. Alcohol and Alcoholism. 2018; 53: 166-172.

[29] Gano A, Pautassi RM, Doremus-Fitzwater TL, Deak T. Conditioned effects of ethanol on the immune system. Experimental Biology and Medicine. 2017; 242: 718-730.

[30] Sapkota M, Wyatt TA. Alcohol, aldehydes, adducts and airways. Biomolecules. 2015; 5: 2987-3008.

[31] Wyatt TA, Gentry-Nielsen MJ, Pavlik JA, Sisson JH. Desensitization of PKA-stimulated ciliary beat frequency in an ethanol-fed rat model of cigarette smoke exposure. Alcoholism, Clinical and Experimental Research. 2004; 28: 998-1004.

[32] Sisson JH. Alcohol and airways function in health and disease. Alcohol. 2007; 41: 293-307.

[33] Simou E, Leonardi-Bee J, Britton J. The effect of alcohol consumption on the risk of ARDS: a systematic review and meta-analysis. Chest. 2018; 154: 58-68.

[34] Zheng Q, Li Y, Zhang L, Yao Q, Zhang J, Li M, et al. Association between drinking and all-cause mortality in patients with ischemic stroke. Nan Fang Yi Ke Da Xue Xue Bao. 2019; 39: 422-427. (In Chinese)

[35] Chiumello D, Busana M, Coppola S, Romitti F, Formenti P, Bonifazi M, et al. Physiological and quantitative CT-scan characterization of COVID19 and typical ARDS: a matched cohort study. Intensive Care Medicine. 2020; 46: 2187-2196.

[36] Lai CC, Shih TP, Ko WC, Tang HJ, Hsueh PR. Severe acute respiratory syndrome coronavirus 2 (SARS-CoV-2) and coronavirus disease-2019 (COVID-19): the epidemic and the challenges. International Journal of Antimicrobial Agents. 2020; 55: 105924.

[37] Plapp BV, Leidal KG, Murch BP, Green DW. Contribution of liver alcohol dehydrogenase to metabolism of alcohols in rats. ChemicoBiological Interactions. 2015; 234: 85-95.

[38] Teschke R. Alcoholic liver disease: alcohol metabolism, cascade of molecular mechanisms, cellular targets, and clinical aspects. Biomedicines. 2018; 6: 106.

How to cite this article: Lin $\mathrm{Lv}, \mathrm{Yi}-\mathrm{Wu} \mathrm{Zhou}$, Rong Yao. Alcohol consumption and COVID-19 severity: a propensity score matched study in China. Signa Vitae. 2021;17(3):112-120. doi: $10.22514 /$ sv.2021.032. 